\title{
Réflexions sur l'improvisation et l'implication sociale de l’éducation musicale. Dialogue avec Jean-Yves Bosseur
}

\author{
Luis Velasco-Pufleau ${ }^{1}$
}

\section{à Henri Pousseur, in memoriam}

Jean-Yves Bosseur (Paris, 1947) est compositeur et musicologue (directeur de recherche au CNRS/ParisSorbonne). Il étudie la composition à la Rheinische Musikschule de Cologne (Allemagne) avec Karlheinz Stockhausen et Henri Pousseur. Compositeur prolifique, son catalogue est constitué d'œuvres de concert (instruments solistes, musique de chambre, orchestre, chœur, dispositif électroacoustique), de créations radiophoniques et d'œuvres ouvertes, événementielles et cinématographiques. Spécialiste de la musique du XXème siècle, il a écrit plusieurs ouvrages sur les rapports de la musique avec les autres arts, en particulier les arts plastiques (www.jeanyvesbosseur.fr).

Ce texte est le fruit d'un échange écrit par courrier électronique, Paris/Bordeaux - en forme de dialogue - et a eu lieu entre 2009 et 2010.

Luis Velasco-Pufleau : La pratique de l'improvisation musicale, dans notre culture musicale occidentale, implique la maîtrise d'un bagage technique et gestuel solide couplée à des capacités poïétiques d'imagination sonore afin de pouvoir créer un objet sonore basé sur un code musical donné. Cette pratique est prédéterminée et façonnée par des années de formation instrumentale, donc une série d'habitudes gestuelles et cognitives ancrées dans le rapport de l'interprète avec son instrument. De ce fait, l'improvisation pure en tant qu'acte spontané et affranchi de toute contrainte préexistante n'existe pas. L'instrumentiste ne peut pratiquement faire que des gestes qu'il maîtrisait auparavant. Néanmoins sa liberté est ailleurs, dans l'agencement et la combinaison de sons dans l'espace temporel, dans la diction et l'énergie mises sur chacun de ses sons, dans l'intention qu'il y met, dans l'instant même de la création, sur chaque partie et sur l'ensemble de l'objet sonore.

Carl Dahlhaus, dans son article « Composition et improvisation », soulevait le paradoxe de l'improvisation en tant qu'acte qui cherche la spontanéité créative mais qui est prédéterminé par des formules répétées et acquises :

Les improvisations qu'on répète cessent par ce fait même d'être des improvisations. [. . .]

L'improvisation repose presque toujours pour une large part sur des formules toutes faites, sur des artifices et des modèles. Le procédé prudent qui consiste à esquisser en pensée le point de départ, les matériaux, le contour ou les articulations d'une improvisation musicale, constitue probablement la règle plutôt que l'exception. Pour ne pas tomber dans l'embarras ou le silence, l'improvisateur doit pouvoir s'appuyer sur un répertoire de clichés disponibles à tout instant, sur un fonds d'éléments préfabriqués qu'il modifie et agence diversement certes, mais qu'il n'invente pas sur le champ. L'idée selon laquelle il s'abandonne sans réserve à la faveur ou à la défaveur du hasard est une fiction. (194)

Cependant, la particularité de l'improvisation ou des formes diverses d'improviser, en tant qu'acte poïétique, repose probablement moins sur le degré de hasard, de nouveauté absolue ou de rupture avec une forme ou des modèles prédéterminés que sur sa capacité à transformer les éléments connus afin de trouver des nouveaux gestes sonores capables de construire de nouvelles structures. Toute création trouve sa liberté dans la contrainte (matérielle, technique, esthétique, spatio-temporelle, rituelle). D'ailleurs, nombreux sont les compositeurs pour qui la rigueur technique était accompagnée d'une pratique de l'improvisation musicale en tant qu'acte poïétique (Bach, Beethoven, Chopin, Debussy, Bartók, Ligeti) et ce fait fut fondamental pour leurs innovations formelles et l'évolution de leurs langages musicaux.

Peut-être faut-il aujourd'hui redéfinir l'objet, les limites et les processus de l'improvisation musicale en tant qu'acte poïétique pour mieux saisir son importance dans le développement de notre culture musicale ?

Jean-Yves Bosseur : Si improvisation signifie composition dans l'instant, collectivement ou non, sans autre donnée et convention préalablement admises que les conditions acoustiques et spatiales de la salle où l'on joue, les exigences et contraintes des instruments ou de la voix, se pénétrer de l'esprit d'improvisation serait alors se mettre, autant que faire se peut, en état de disponibilité, prêt à capter tout événement et à y réagir créativement, à l'affût. Approfondir de telles qualités, liées à la concentration sur le moment, se révélera, sans aucun doute, très précieux pour le musicien, à la fois pour l'apprentissage de sa pratique et pour des activités qui s'adressent en priorité à son imagination, se situant quelque part sur cet axe dont les deux pôles, détermination/indétermination représentent des entités virtuelles, illusoires, plus proches, toutefois, du second terme que du premier. D'ailleurs, comme le déclare François Jeanneau, « la composition vient de l'improvisation et y retourne ». Le « et y retourne » fait précisément toute la différence ; l'écrit n'est pas considéré comme la 
finalité de l'acte de création : il peut être utile comme moyen mnémotechnique, puis s'effacer à nouveau pour laisser s'imposer un mode de communication musicale qui le dépasse.

Dans son acception actuelle, l'improvisation ne saurait être, à mon sens, schématiquement opposée à l'écrit, en raison de la variété des modes de notation expérimentés au cours des dernières décennies ; peut-être pourra-telle se ranger aux côtés des musiques variables, qui appellent justement d'autres formes d'écriture, stimulations graphiques ou verbales qui contribuent à faire éclater la notion d'œuvre-objet pour l'orienter plutôt vers celle de processus ouvert. «Ce qui m'intéresse, écrit Luc Ferrari, c'est une subversion de l'écriture. C'est dans ce sens que la simplicité est subversive. Expérimentation d'une nouvelle simplicité ; pas de camouflage possible ; les portes sont ouvertes à l'invention spontanée. Ainsi peut apparaître une autre complexité qui n'est pas en façade, mais que l'on doit aller chercher en profondeur, à l'intérieur du moment » (cité dans Bosseur, La musique du XXe siècle 230).

Tenter de cerner le concept d'improvisation me paraît tout à fait paradoxal, car l'improvisation n'est pas un lieu dont les frontières sont clairement délimitées au sein des pratiques musicales. L'improvisation est un phénomène excessivement fragile, tout d'abord parce que, en raison de sa nature même, il s'agit d'une pratique vouée à l'éphémère, au fugitif, qui implique des remises en question et en tension permanentes ; ensuite, parce que nous devons tenter d'appréhender son essence par delà ou en marge des critères de valeur drainés par l'écriture, alors que notre apprentissage occidental est précisément fondé presque entièrement sur les lois et les vertus de l'écrit.

L'improvisation a pour mission de révéler ce qui déborde la conscience réfléchie, le savoir et la mémoire, ce qui surgit avant que la pensée logique ne s'en empare, dans une sorte d'urgence, avec un dynamisme énergétique intraduisible selon les normes de l'écriture. Si n'importe quoi doit pouvoir survenir, il s'agirait alors, selon Bernard Lubat, d'un n'importe quoi « qui d'un coup contourne la pensée, casse la carapace, laisse s'insinuer la révolte qui pousse à fonctionner sans savoir. Attention, faire n'importe quoi, c'est très dur, parce qu'il faut avoir la rigueur de reconnaître de quoi il est question » (Levaillant 31). Faute de quoi, l'improvisation risque bien de n'être qu'une accumulation amorphe de clichés individuels, réflexes instrumentaux et habitudes, lieux communs déduits de modèles écrits, parfois simplement caricaturés, résidus de formes prédigérées, trop de fois ressassées, ou encore démonstration narcissique complaisante d'un savoir-faire ou d'une habileté de virtuose.

Luis Velasco-Pufleau : Bien que le concept d'improvisation musicale soit difficile à définir par sa nature polymorphe et ses nombreuses pratiques, le geste sonore qui est à la base de toute improvisation - celui qui cherche à traduire un son interne dans une sonorité externe produite par la voix ou par un instrument - mériterait toute notre attention et notre réflexion. En effet, d'un point de vue poïétique, même avant de devenir geste, les sons internes du musicien reflètent un monde pré-rationnel dans lequel se trouvent les fondements de la création sonore. Puis, le musicien ordonne temporellement ces sons dans un langage spécifique qui est en accord avec une culture et un moment historique donné. Ainsi, l'improvisation serait à la base du développement des langages musicaux de même que dans la disponibilité intuitive à réagir de façon sonore dans l'instant créatif.

En approfondissant cette réflexion poïétique, la différenciation d'une improvisation idiomatique d'une autre à caractère non-idiomatique se révèle pertinente. L'improvisation idiomatique étant celle qui fait référence à un langage musical particulier, avec une grammaire et une syntaxe établies. L'improvisation non-idiomatique étant celle qui construit son discours sur d'autres paramètres du son, comme la texture, la couleur et le timbre, ou en utilisant des références symboliques du réel, imaginatives ou concrètes, tels que les émotions, le visuel, ou tout élément de la réalité. Ces deux sortes d'improvisation se différencient du point de vue poïétique par les différents degrés de rationalité et d'abstraction qu'elles engagent, sans pour autant s'exclure l'une de l'autre. L'une pourrait être évaluée principalement par la maîtrise du code, l'autre par sa puissance poétique. Sur ce point spécifique, la poïétique de l'improvisation musicale serait-elle rapprochée de celle des autres systèmes symboliques, comme le langage ou les autres arts?

Jean-Yves Bosseur : Je n'ai jamais souhaité mettre en parallèle de manière par trop systématique la musique et les autres disciplines artistiques, même si j'ai, à plusieurs reprises, cherché à sonder les modalités d'articulation que l'on pouvait développer entre elles. Je considère en effet que la pratique musicale possède une logique qui lui appartient en propre, et qui ne saurait être évaluée par rapport à d'autres contextes de communication artistique. J'ajouterai que cela me semble d'autant plus manifeste dans le cas de l'improvisation, qui défie les codes de l'écrit. Je me pose par ailleurs des questions sur la différenciation que vous opérez entre improvisations idiomatique et non-idiomatique. À mon sens, même lorsque l'on ne peut déceler une référence à un langage en particulier, l'improvisation apparaît nourrie des explorations et expérimentations que l'on découvre dans différentes tendances de la musique contemporaine, et réciproquement. Travailler sur les notions de texture, de couleur et de timbre, renvoie très certainement à de nouveaux idiomes (développés notamment par Ligeti, Scelsi, Xenakis, Nono...) qui, certes, ne constituent pas un système aussi régulateur que celui de la tonalité ou de la modalité, mais traduisent des options esthétiques et formalisatrices tout à fait identifiables, véritables jalons ou pistes de réflexion pour de nouvelles approches de l'improvisation, qu'il me paraît difficile d'ignorer aujourd'hui en tant que musicien, même s'il est hors de question de les utiliser en tant que modèles. 
Luis Velasco-Pufleau : Certes, l'apport des compositeurs de la deuxième partie du XX ${ }^{\text {ème }}$ siècle, de même que celui du jazz et de certaines musiques de traditions extra-européennes, a été fondamental pour le développement de la pratique de l'improvisation musicale. La réciprocité est aussi incontestable. Ce point éveille en moi deux questions par rapport à la différenciation et à l'interdépendance entre indétermination, formes mobiles et improvisation musicale.

Aujourd'hui nous pouvons considérer les différentes formes qu'a pris l'indétermination comme l'un des changements les plus profonds dans la théorie et le langage musical occidental, dès la fin de la Deuxième guerre mondiale jusqu'à nos jours (depuis Cage, Feldman et Brown jusqu'aux compositeurs contemporains, vous-même inclus, en passant par Stockhausen, Pousseur, Boulez, Berio, Cardew, Ligeti, Scelsi, etc.). Ce changement a non seulement bouleversé les fondements et les principes du système musical occidental (tels la forme, la notation, le temps, la hauteur, le rythme, le timbre, la texture, l'espace sonore), mais a aussi brouillé les frontières et les rapports entre objet sonore, compositeur, interprète et auditeur. De quelle façon la pratique de l'improvisation a-telle contribué à l'avènement de ce bouleversement ? Considérez-vous que les formes mobiles et l'indétermination constituent une sorte d'improvisation contrôlée ?

Jean-Yves Bosseur: Non, je dirais plutôt que l'improvisation d'une part et le recours à de nouvelles formes de notation et de communication, plus libres que celles que nous avons pratiquées jusque là d'autre part, représentent deux manières relativement autonomes de mettre en évidence la personnalité individuelle des musiciens. Elles peuvent se nourrir l'une de l'autre, se rapprocher, sans pour autant se confondre. Parmi les différences majeures que l'on peut observer entre ces deux voies, il y en a tout simplement au moins deux : la présence d'une partition, même si celle-ci se réduit à quelques graphismes ou à un scénario verbal, ainsi que le fait qu'elle sous-entende toujours l'existence d'un compositeur, qui demeure l'initiateur de l'événement musical. Historiquement, on pourrait d'ailleurs constater que ces deux types de pratique se sont développées quasi simultanément avec, comme je le disais précédemment, certains entrecroisements, telle la participation du saxophoniste de jazz Steve Lacy au sein du groupe Musica Elettronica Viva vers la fin des années 60. L'avènement du free jazz constitue de son côté une sorte de critique des structures traditionnelles du jazz, mais certainement pas de la musique savante occidentale, avec lequel il entretient en définitive assez peu de relations. Certes, ces deux pratiques, l'une de nature orale, l'autre qui continue à se servir de certains principes de notation, n'ont cessé de s'observer mais, je pense, tout en préservant une relative distance entre elles. Cela me rappelle une discussion que j'ai eue avec Earle Brown en 1999 :

Je travaillais à Folio quand Pierre Boulez est venu à New York pour la première fois en tant que chef, avec la compagnie Jean-Louis Barrault. II a regardé la partition et nous avons discuté à propos de la forme ouverte. Lisez ce que j'ai écrit à cet égard avant que Pierre publie son article « Aléa ». Je ne voudrais pas paraître trop égocentrique, mais ce qui est historiquement vrai, c'est qu'il a écrit cet article en 1957, après que je lui ai rendu visite à Paris, en 1956. Un certain nombre de ces idées sur l'improvisation guidée, qu'il développe dans son article sont directement venues de notre conversation. Pierre aimait beaucoup les Trois Pièces pour piano, Perspectives, Music for Cello and Piano, parce qu'elles ressemblaient à sa propre musique. Je lui ai montré Folio et il était très réticent, considérait que c'était une mauvaise idée. Nous sommes les compositeurs, et c'est nous qui sommes détenteurs du bon goût. Nous savons ce qu'il faut faire. Les musiciens, ce sont des travailleurs, en définitive. II ne partageait pas mes vues. John Cage non plus, d'ailleurs, n'adhérait pas à mon intérêt pour la flexibilité et l'improvisation, qu'il n'a jamais appréciée. Quand j'ai composé December 52 et lui en ai expliqué le principe, il m'a dit: "Tu vas les laisser faire, ils vont juste jouer leurs petits airs favoris ». Je lui ai répondu: «Non, je ne le pense pas ; je ne vois pas comment ils pourraient justifier de jouer de telles choses, compte tenu de la nature abstraite du graphisme que je leur propose. Ils doivent jouer des structures de son de type abstrait plutôt que des mélodies, parce que rien ne justifie une tonalité de do majeur dans cette page ॥. II n'avait pas confiance dans l'improvisation, parce qu'il pensait que cela risquait de provoquer de l'auto complaisance de la part des musiciens. Cela pourra paraître aussi un peu narcissique, mais j'ai toujours pensé que je n'avais pas eu une bonne influence sur John. Quand je l'ai rencontré, il composait des partitions très strictes, à base d'opérations de hasard, jets de dés et de pièces de monnaie ; les interprètes jouaient le résultat de ces procédés de hasard, de manière aussi précise que possible, aussi exactement qu'ils l'auraient fait avec la musique de Mozart. C'est seulement la partition qui était composée à partir du hasard. II n'était pas permis d'être spontané, de s'adonner à l'improvisation. Un peu plus tard, comme il en a témoigné dans ses livres, il s'est intéressé à l'indétermination, et la première pièce dans cette voie a été le Concert de piano (1957-1958). Et laisser les musiciens effectuer des choix dans sa musique lui a posé bien des problèmes. On peut observer une rupture entre les pièces de hasard de John, qui ont des qualités particulières et, après cela, celles où il a perdu le contrôle. Parce que John n'a jamais été, comme moi, un interprète au sein d'un orchestre, ou dans une formation de jazz. (Bosseur, De vive voix 95-97)

En effet, les notations graphiques inventées par Brown, par exemple dans December 52, sont en partie venues de l'insatisfaction provoquée par sa propre pratique de l'improvisation dans des groupes de jazz, en milieu universitaire. II a alors cherché à désorienter les musiciens, à les faire sortir de leurs réflexes plus ou moins conditionnés, et c'est par le biais de stimulations graphiques qu'il l'a fait. 
Pour ma part, je travaille depuis peu avec un petit groupe d'instrumentistes improvisateurs réunis par Benjamin Bondonneau, auquel se joint occasionnellement le chanteur basque Benat Achiary. Bondonneau m'a contacté parce qu'il avait eu connaissance de mon livre-partition Le temps de le prendre [Paris : Kimé, 1997], où j'ai rassemblé de nombreux textes à la lisière de la pratique de l'improvisation. Cela montre bien que ces deux domaines d'activité musicale ne sont nullement étanches et tout à fait susceptibles de dialoguer. Encore faut-il se garder de toute forme de confusion et bien prendre en compte le passé musical des personnes concernées, certaines étant avant tout instrumentistes (comme Derek Bailey, Joëlle Léandre...), même si l'acte de composition ne leur est pas étranger, d'autres ayant plutôt choisi de s'exprimer en tant que compositeurs. Je considère que c'est mon cas, car cela fait très longtemps que j'ai abandonné mon instrument, l'alto, ne me sentant pas suffisamment capable de me livrer « dans l'instant », à la différence de mes amis improvisateurs.

Luis Velasco Pufleau : Il existe actuellement des groupes d'improvisation et de recherche, tels la International Society of Improvisation Music ${ }^{2}$ et le projet Improvisation, Community and Social Practice ${ }^{3}$, qui voient dans l'improvisation musicale un modèle de relations sociales et de dialogue politique entre différentes cultures ${ }^{4}$. La pratique de l'improvisation serait politique par sa puissance à redéfinir les rapports sociaux à l'intérieur du groupe. Le développement de l'individu par la liberté créative, le partage des responsabilités et l'échange collectif sont à la base de cette conception de la pratique musicale. Dans la musique savante, un exemple représentatif de cette idée est le parcours atypique du compositeur et improvisateur Cornelius Cardew (1936-1981). Quelle est votre position envers cette conception de la pratique de l'improvisation ?

Jean-Yves Bosseur : Je m'en sens totalement complice. À condition toutefois de ne pas isoler l'improvisation en tant que catégorie à part. Comme je l'ai dit précédemment, selon moi, il n'y pas de raison de couper une telle pratique de certaines formes de transmission musicale " ouvertes ", qui intègrent des éléments de notation (graphiques, verbales...). Pour en revenir plus explicitement à votre question, il est indéniable que l'improvisation possède de fortes connotations politiques, notamment les hiérarchies généralement confirmées par l'enseignement académique entre les compositeurs et les exécutants, entre les musiques reposant sur les principes de l'écriture et celles qui ne passent pas par un tel filtre, entre les instrumentistes qui ont suivi l'apprentissage des conservatoires et les autres... Plus que dans tout autre type d'engagement, de nature intrinsèquement politique, c'est bien dans celui-ci que je me suis toujours senti concerné. En effet, en tant que musicien, il me semble que je comprends tout à fait ses enjeux, alors que les diktats des différents partis politiques me laissent généralement pour le moins perplexe. Il est intéressant que vous mentionniez à ce propos Cornelius Cardew. Je l'ai un peu fréquenté, au tout début des années 70, et nous avons même assisté ensemble, à Londres, à un concert du Scratch Orchestra, dont la démarche m'intéressait alors au plus haut point. Peu de temps après, le groupe dont je faisais partie à cette époque, le Groupe d'Étude et de Réalisation Musicales, avait créé une revue, Germinal, qui accordait une large place à la création collective. Nous souhaitions consacrer un numéro aux activités du Scratch Orchestra en publiant une traduction d'un certain nombre de textes, propositions musicales... Fondé à Londres en 1969 par Michael Parsons, Howard Skempton et Cornelius Cardew, le Scratch Orchestra consistait, à l'origine, en un projet réunissant une cinquantaine de musiciens, professionnels ou amateurs, qui décidèrent de mettre en commun leurs ressources musicales, tout en préservant une large part d'initiative personnelle et de libre expression. À partir d'un certain nombre de principes exposés dans la "Draft Constitution", chaque membre de l'orchestre tenait notamment une sorte de journal, le Scratch Book, où étaient consignés, selon des modes de notation diversifiés, projets de pièces, idées de concert, rituels d'improvisation, arrangements de musiques pré-existantes, le but étant que chacun écrive un nombre de pièces égal ou supérieur à l'effectif de l'orchestre. Le groupe parvint ainsi à allier écriture et improvisation et à associer différents types de musiques. De 1969 à 1971, plusieurs membres de ce groupe, qui eut une indéniable portée didactique, prirent, à tour de rôle, la responsabilité d'un concert, en puisant dans un répertoire de catégories musicales préalablement définies.

L'activité de cette communauté musicale cesse pourtant assez rapidement, à la suite de prises de position de caractère politique de John Tilbury et de Cornelius Cardew en 1971; l'ouvrage Stockhausen serves imperialism (Latimer Press, Londres, 1974) $)^{5}$ de C. Cardew témoigne très clairement de la distance radicale adoptée par certains musiciens, décidés à engager leur action dans le sens d'une démarche politique, vis-à-vis de ce qu'il est convenu d'appeler l'avant-garde. Pour C. Cardew, en effet, l'aspect subversif du Scratch Orchestra, par rapport aux institutions, demeure chimérique parce que son activité est coupée de la réalité sociale et de ses luttes, de toute conscience de classe; le Scratch perpétue le courant de l'art pour l'art, réservé à l' "establishment", en une pratique qui se veut au-dessus des classes sociales; à travers un nihilisme apparent, ses principes jouent le jeu du libéralisme dont il représente en définitive une forme de cautionnement. Dès 1971, J. Tilbury dénonce donc l'impasse du groupe et, plus globalement, la paralysie de l'idéalisme bourgeois, représenté par les anarchistes et les libéraux; avec C. Cardew, il fonde l'Ideology Group qui réfléchit, à partir de principes d'analyse marxistes, sur les rapports entre la classe prolétarienne et la musique, envisageant de quelle manière celle-ci peut participer d'un même élan révolutionnaire. Stockhausen serves imperialism contient également une autocritique sans complaisance de ses propres œuvres.

Ignorant tout du virage idéologique radical de Cardew, je lui ai donc téléphoné pour lui demander son autorisation à propos des textes du Scratch Book. II m'a répondu assez froidement que nous pouvions bien faire ce que nous voulions, car il s'était définitivement écarté de cette voie. Ce n'est qu'un peu plus tard, en lisant 
Stockhausen Serves Imperialism, que j'ai appris qu'il avait fait le choix d'un engagement explicitement politique, d'obédience maoïste. J'avoue n'avoir jamais vraiment adhéré à ses prises de position, même si certaines posent des questions souvent pertinentes sur la place de l'avant-garde dans notre société et qui demeurent du reste, près de quarante ans après, tout à fait actuelles.

Pour ma part, beaucoup plus simplement, j'ai eu tendance à estimer que c'était au sein même de mon activité musicale que j'étais en mesure de prendre position, en dénonçant notamment les hiérarchies et privilèges évoqués précédemment ; la transgression de la notion d'œuvre d'art, des pouvoirs de l'écriture aussi bien que des illusions technologiques, me paraissait pouvoir constituer une réponse tangible à un tel débat.

Luis Velasco Pufleau : Je suis d'accord avec vous dans l'idée que l'improvisation possède des fortes connotations politiques dans notre société où l'écriture a une place prépondérante. La transgression des hiérarchies sociales passerait par un changement profond des pratiques musicales : un décloisonnement des frontières établies par un ordre social et politique basé sur le respect d'un canon esthétique que certaines institutions imaginent conserver. En sacralisant la partition, ces institutions acceptent difficilement l'inévitable changement des notions d'interprétation, de production, d'écoute et de médiation musicale. Pour accomplir ce dessein, la pédagogie musicale a un rôle fondamental à jouer car ces frontières se mettent en place dès le début de l'enseignement musical. En effet, une pratique musicale orale, fondée sur l'exploration sonore de la voix et de l'instrument, peut constituer une base fertile préalable à l'apprentissage de la lecture. Puis, le développement des capacités nécessaires pour la pratique de l'improvisation peut se mener, au sein d'un groupe et avec l'aide de méthodes spécifiques ${ }^{6}$, en même temps que l'acquisition d'une maîtrise de la théorie et de l'écriture musicale. Sur ce point, la pratique collective renforce la notion de partage et d'entraide par opposition à une pratique solitaire qui chercherait une réussite exclusivement individuelle. Enfin, l'apprentissage de l'interprétation des différents genres musicaux ne peut que gagner avec l'ouverture stylistique de l'éducation musicale collective. Pour cela, la pratique personnelle des professeurs doit aller de pair avec leur travail pédagogique. L'arrêt de l'opposition entre composition et improvisation, entre oralité et écriture, en est une condition nécessaire pour bénéficier de leurs richesses complémentaires. Car, citant à nouveau Carl Dahlhaus, " ce n'est pas dans la dissolution de la composition dans l'improvisation [ou l'inverse], mais bien de la médiation entre elles qu'on peut attendre quelque chose de pertinent » (199).

Pour conclure, quels sont les changements que vous croyez nécessaires dans l'enseignement afin de renforcer le rôle de la musique dans la transformation sociale et dans le développement de l'individu au sein d'un monde multiculturel?

Jean-Yves Bosseur : Il est incontestable que la pratique de l'improvisation contient en filigrane de précieux indices sur les hypothèses de relation au sein d'un groupe, avec les incidences socio-politiques que cela implique. Elle pourrait même être en mesure d'avancer des sortes de modèles de comportement humain, par delà les rapports de force que nous ne connaissons que trop, jusque dans le langage artistique. Peut-être une telle prise de conscience explique-t-elle d'ailleurs la méfiance dont elle fait l'objet de la part des « officiels » de la musique. II est tellement commode, en en caricaturant les modalités de fonctionnement, de la considérer avant tout comme la conséquence de l'esprit soixante-huitard!

Je souhaiterais citer ici les propos de mon maître Henri Pousseur, à qui ce texte est dédié :

Je vois tout particulièrement dans le développement de ce que l'on a appelé les "formes ouvertes" la prochaine étape d'une série de mutations ou sauts qualitatifs, devant ou du moins pouvant nous conduire à la constitution d'une nouvelle cohérence de langue musicale, même plurielle. En ouvrant précisément le champ aux capacités d'invention collective, il allait permettre les premiers pas d'un dépassement de l'excessif négativisme qui avait régné jusque-là dans la musique d' "avant-garde" et atteint des extrémités parfois proches de l'étranglement. En appelant de plus en plus clairement à une remise à l'honneur de l'improvisation (mais au sein d'une conception qui accepte tous les degrés de préparation réfléchie aussi bien que de spontanéité immédiate, où l'écrit même le plus précisément exigeant joue son rôle d'auxiliaire), il suggérait l'éclatement des parois fort étanches par lesquelles cette musique s'était toujours plus sévèrement refermée sur elle-même, et le rétablissement de communications et d'échanges avec d'autres, finalement avec toutes les autres formes d'existence musicale (Pousseur 5).

II me semblerait indispensable que les Conservatoires accordent une place plus importante à la pratique collective, à la fois à travers l'approche de l'improvisation et des partitions de forme ouverte, ces deux aspects pouvant se conjuguer avec bonheur, et la phrase de Dahlhaus que vous citez me paraît particulièrement éloquente. À ce propos, j'évoquerais volontiers la classe d'Improvisation Générative créée au CNSM de Paris par Alain Savouret et Rainer Boesch. Alain Savouret vient d'ailleurs de publier aux éditions Symétrie un ouvrage remarquable, Introduction à un solfège de l'audible : L'improvisation libre comme outil pratique, dans lequel il relate précisément ses conceptions et expériences en ce domaine. J'ai eu à plusieurs reprises l'occasion de faire partie du jury du concours de fin d'année de cette classe et ai toujours été fortement impressionné par la force de 
conviction et l'inventivité de certains des étudiants qui avaient choisi de suivre ce cursus. Certes, ce type de musique demeure encore largement ignoré par les grandes instances spécialisées dans la musique contemporaine, tels Musica à Strasbourg et Ars Musica à Bruxelles, mais on ne peut que se réjouir du développement de plusieurs festivals, par exemple à Marseille et à Vandoeuvre, qui soutiennent avec vigueur ce type d'activité musicale, sans nécessairement l'enfermer dans un ghetto, en le séparant des formes d'écriture dont j'ai parlé précédemment. II y a quelques années, j'avais notamment présenté une version d'extraits des Song Books de Cage, avec la chanteuse Hélène Ruggeri, et l'accueil avait été des plus chaleureux. Sans être improvisateur moi-même, faute d'une compétence instrumentale suffisante, je considère comme infiniment précieuses ces facultés d'échange entre l'écrit et l'oral, avec la chance que cela peut procurer de travailler avec des musiciens qui ne correspondent pas forcément aux critères de sélection académiques.

Pour conclure, une courte anecdote très significative : il y a de cela plus d'une trentaine d'années, j'avais rencontré Archie Shepp, en vue d'un concert organisé par la Maison de la Culture de la Corse à Ajaccio ; on m'avait juste demandé de prendre contact avec lui pour organiser l'événement. À l'époque, je jouais encore de l'alto et venais d'une répétition avec mon groupe. Shepp me dit alors : « Tu vas jouer avec nous, toi ». Et je lui répondis que je n'avais que fort peu d'expérience de l'improvisation de jazz. Il ajouta : " Mais on ne va pas faire du jazz, on va faire de la musique ». Face à un tel défi, que pouvais-je répliquer ? Et le concert auquel j'ai fini par participer, avec un incontestable plaisir, reste un moment inoubliable...

\section{Notes}

${ }^{1}$ Docteur en Musique et musicologie (Université Paris-Sorbonne), Luis Velasco-Pufleau est post-doctorant au Centre de Recherches sur les Arts et le Langage (CRAL) de l'École des Hautes Études en Sciences Sociales (EHESS, Paris). Ses recherches portent sur les liens entre musique et politique au XXème siècle, sur les rapports entre le compositeur et l'interprète dans la création musicale contemporaine et sur l'interprétation et l'improvisation musicales. Il est chargé de cours en musicologie à l'Université de Bordeaux (www.luisvelascopufleau.com).

\section{${ }^{2}$ www.isimprov.org/index.html}

${ }^{3}$ www.improvcommunity.ca

${ }^{4}$ L'idée que la pratique musicale peut fournir un modèle d'interaction sociale est développée par le sociologue Alfred Schütz dès le début des années 1950 (« Making music together. A Study in Social Relationship »).

${ }^{5}$ En ligne sur l'adresse : http://www.ubu.com/historical/cardew/cardew stockhausen.pdf

${ }^{6}$ Un exemple réussi en est le livre de Edward Sarath, Music Theory Through Improvisation. A New Approach to Musicianship Training.

\section{Bibliographie}

Bosseur, Jean-Yves. La musique du XXe siècle à la croisée des arts. Paris: Minerve, 2008. Imprimé

---. De vive voix : dialogues sur les musiques contemporaines. Paris : Minerve, 2010. Imprimé.

Dalhaus, Carl. « Composition et improvisation ». Essais sur la Nouvelle Musique. 1972. Genève : Contrechamps, 2004. 191-199. Imprimé.

Levaillant, Denis. L'improvisation musicale. Paris : Lattès, 1981. Imprimé.

Pousseur, Henri. «Musique moderne, musique pédagogique, pédagogie musicale ». Les musiques de notre temps - création, enseignement, diffusion. Strasbourg : Conseil de l'Europe, 1985. 7. Imprimé.

Sarath, Edward. Music Theory Through Improvisation: A New Approach to Musicianship Training. New York: Routledge, 2010. Imprimé.

Savouret, Alain. Introduction à un solfège de l'audible : L'improvisation libre comme outil pratique. Lyon : Editions Symétrie, 2011. Imprimé. 
Schütz, Alfred. «Making Music Together: A Study in Social Relationship ». Collected papers. Studies in Social Theory. Ed. Arvid Brodersen. Vol. 2. The Hague: Martinus Nijhoff, 1964. 159-178. Imprimé. 